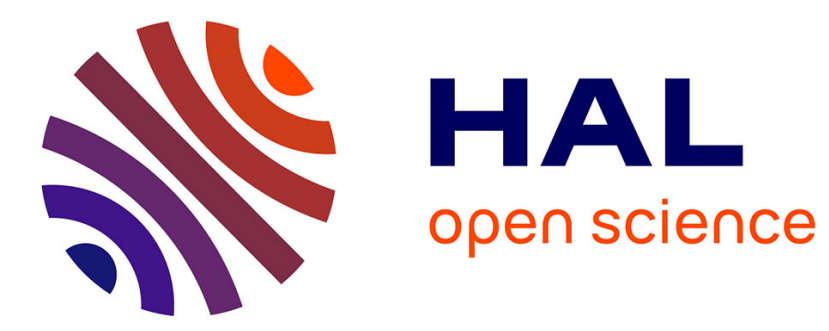

\title{
The PKC- $\zeta$ interacting protein ZIP3 is generated by intronic polyadenylation and is expressed in brain and retina of the rat
}

\author{
Andreas Urbanczyk, Anselm Jünemann, Ralf Enz
}

\section{- To cite this version:}

Andreas Urbanczyk, Anselm Jünemann, Ralf Enz. The PKC- $\zeta$ interacting protein ZIP3 is generated by intronic polyadenylation and is expressed in brain and retina of the rat. Biochemical Journal, 2010, 433 (1), pp.43-50. 10.1042/BJ20101111 . hal-00547993

\section{HAL Id: hal-00547993 \\ https://hal.science/hal-00547993}

Submitted on 18 Dec 2010

HAL is a multi-disciplinary open access archive for the deposit and dissemination of scientific research documents, whether they are published or not. The documents may come from teaching and research institutions in France or abroad, or from public or private research centers.
L'archive ouverte pluridisciplinaire HAL, est destinée au dépôt et à la diffusion de documents scientifiques de niveau recherche, publiés ou non, émanant des établissements d'enseignement et de recherche français ou étrangers, des laboratoires publics ou privés. 


\title{
The PKC- $\zeta$ interacting protein ZIP3 is generated by intronic polyadenylation and is expressed in brain and retina of the rat
}

\author{
Andreas Urbanczyk *, Anselm Jünemann † and Ralf Enz* \\ * Institut für Biochemie (Emil-Fischer-Zentrum) \\ Friedrich-Alexander-Universität Erlangen-Nürnberg, 91045 Erlangen, Germany \\ † Augenklinik mit Poliklinik \\ Friedrich-Alexander-Universität Erlangen-Nürnberg, 91054 Erlangen, Germany
}

\begin{abstract}
Running Title: Expression of ZIP3 in human and rat CNS
Correspondence to: Dr. Ralf Enz, Institut für Biochemie Friedrich-Alexander-Universität Erlangen-Nürnberg

Fahrstr.17, D-91054 Erlangen, Germany

Phone: (+49) 9131/85-26205

Fax: (+49) 9131/85-22485

Email: ralf.enz@biochem.uni-erlangen.de
\end{abstract}

\begin{abstract}
Abbreviations: CNS - central nervous system, DAPI - 4,6-diamidino-2-phenylindole, GABA receptor - $\gamma$-aminobutyric acid type C receptor, GAPDH - glyceraldehyde-3-phosphate dehydrogenase, HEK-293 cells - human embryonic kidney cells, INL - inner nuclear layer, $\mathrm{IPL}$ - inner plexiform layer, GCL - ganglion cell layer, ONL - outer nuclear layer, OPL - outer plexiform layer, PB1 domain - Phox and Bem1p1 domain, PKC - protein kinase C, PRS photoreceptor segments, ZIP - PKC- $\zeta$ interacting protein, UBA domain - ubiquitin associated domain
\end{abstract}




\section{ABSTRACT}

Scaffold proteins contain multiple protein-protein interaction modules that physically assemble functional related proteins into larger complexes. Protein kinase $\mathrm{C}-\zeta$ interacting proteins (ZIP) link the enzymatic activity of the atypical protein kinase $C$ isoforms $P K C-\lambda / \iota$ or PKC- $\zeta$ to target proteins and are associated with neurodegenerative disorders. In the rat, alternative splicing generates 3 ZIP variants. Previously, we identified the ZIP3 transcript containing $13 \mathrm{C}$-terminal amino acids encoded by intron 4 in the rat central nervous system (CNS). Here, we identified intronic polyadenylation signals in rat and human ZIP genes and detected the corresponding ZIP3-like transcripts. In addition, we generated ZIP3 specific immunesera and observed expression of the protein in brain and retina of the adult rat. In the retina, ZIP3 is present in nuclear layers where it co-localizes with PKC- $\zeta$. An immuneserum recognizing all 3 ZIP isoforms labelled the same cells as the newly generated ZIP3 specific antibodies and in addition stained both synaptic layers of the retina. There, ZIP proteins are localized in axon terminals of rod bipolar cells that also contain ZIP-interacting PKC- $\zeta$ and $\mathrm{GABA}_{C}$ receptors. In summary, we detected ZIP3-like transcripts in rat and human and describe the expression of ZIP3 in the rat CNS.

Keywords: ZIP3, p62, sequestosome-1, SQSTM1, GABA ${ }_{C}$ receptor, retina 


\section{INTRODUCTION}

The correct targeting, localization and regulation of kinases and phosphatases represent major mechanisms underlying the complex function of intracellular signal cascades. These tasks are accomplished by the formation of signal complexes that use multidomain protein adaptors as scaffolds to physically link functionally related proteins. The family of protein kinase $\mathrm{C}-\zeta$ interacting proteins (ZIP) are typical scaffolds that ensure the vicinity of the enzymatic activity of the atypical protein kinase $C$ isoforms PKC- $\lambda / \iota$ or PKC- $\zeta$ to target proteins [1]. While the abbreviation ZIP is used for the rat proteins, homologous proteins in humans are designated p62 or sequestosome-1 and A170/STAP is used for the mouse counterparts [2]. For the sake of clarity, in this manuscript we will use the term ZIP for all orthologues.

ZIP proteins participate in a number of intracellular signal cascades, many of them regulating NF-KB activity [3]. While ZIP-deficient mice suffer from insulin resistance and obesity [4], proteins of the ZIP family are also present in the central nervous system (CNS) and have been linked to neurodegenerative disorders, such as Alzheimer's and Parkinson's disease and dementia with Lewy bodies $[5,6]$. Interestingly, ZIP1 acts as a chaperone preventing aggregation of the hyperphosphorylated tau-protein in Alzheimer's disease and indeed, ZIP-deficient mice show Alzheimer-like neurodegeneration in the hippocampus and cortex [7]. ZIP proteins might also be involved in neuronal excitability, as suggested by their direct interaction with ion-channels and receptors in the CNS, including auxiliary subunits of voltage-gated potassium channels $(K v \beta 2)$, the $D_{2}$ dopamine receptor and $G A B A_{C}$ receptors [8-10].

ZIP proteins contain multiple protein-protein interaction sites [11]. In ZIP1, a N-terminal PB1 domain is followed by a ZZ zinc-finger, several linear binding motifs, a serine/threonine rich region and an UBA domain. The PB1 domain of ZIP proteins can dimerize or bind to the PB1 domain of the atypical protein kinases PKC- $\zeta[11,12]$. ZIP2 lacks the TRAF6 binding motif present in ZIP1 by skipping of exon 5 [9]. In rat, we identified a third ZIP isoform (ZIP3) that is characterized by an alternative C-terminus following exon 4 and thus contains only the PB1 and ZZ zinc-finger domains [8]. However, so far a ZIP3 transcript was detected in the rat only and the existence of the corresponding protein has not been demonstrated due to the lack of ZIP3 specific antibodies.

Here, we compared intronic sequences between rat, mouse and human ZIP genes and identified alternative polyadenylation signals in intron 4 of rat and human that result in ZIP3like transcripts. Furthermore, we generated ZIP3 specific immunesera and observed the expression of the ZIP3 protein in brain and retina of rat. Finally, we compared the localization of ZIP3 with other ZIP proteins and ZIP interaction partners in the rat retina.

\section{EXPERIMENTAL}

\section{Sequence Analysis}

Genomic sequences of ZIP isoforms from rat, mouse and human were taken from www.ncbi.nlm.nih.gov/gene. Splice donor and acceptor sites were analyzed with www.fruitfly.org/seq_tools/splice.html and polyadenylation signals were predicted manually according to the consensus sequences AAUAAA and AUUAAA [13].

\section{Isolation of RNA and RT-PCR}

Total RNA was extracted from brain of adult rats, mice or from one $10 \mathrm{~cm}$ dish of HEK-293 cells, reverse transcribed and amplified as described [8]. Incubation times for cDNAsynthesis were $10 \mathrm{~min}$ at room temperature followed by $50 \mathrm{~min}$ at $42{ }^{\circ} \mathrm{C}$ and $10 \mathrm{~min}$ at $70{ }^{\circ} \mathrm{C}$. For PCR amplification the following parameters were used: $94{ }^{\circ} \mathrm{C}$ for $3 \mathrm{~min}$ followed by 28 cycles at $94{ }^{\circ} \mathrm{C}$ for $45 \mathrm{~s}, 60^{\circ} \mathrm{C}$ for $45 \mathrm{~s}, 72{ }^{\circ} \mathrm{C}$ for 2 min and a final incubation at $72{ }^{\circ} \mathrm{C}$ for $10 \mathrm{~min}$. To compare amounts of isolated RNA and the efficiency of reverse transcription 
between samples, a PCR with oligonucleotides recognizing GAPDH (Tab. 1) was performed for 25 cycles with the parameters described above. Controls were treated as above without adding reverse transcriptase. All PCR products were identified by DNA sequencing.

\section{Generation of ZIP3 specific antibodies}

A peptide containing $13 \mathrm{C}$-terminal amino acids specific for the rat ZIP3 isoform was $\mathrm{N}$ terminally extended by two amino acids $(E, S)$ that are conserved in all 3 ZIP isoforms to enhance the immune response. In addition, a N-terminal cysteine was used for coupling to keyhole limpet haemocyanin. Peptide synthesis, immunization of rabbits and guinea pigs and affinity purification of resulting immunesera was accomplished by a company (Pineda Antikörper-Service, Berlin, Germany).

\section{Protein expression in E.coli and Western blotting}

Coding sequences of ZIP1, ZIP2 and ZIP3 from rat were N-terminally tagged with the T7epitope by cloning in pET-21a (Novagen, Madison, WI) and expressed in E.coli as described [8]. Obtained lysates were subjected to SDS-PAGE and analyzed by Western blotting using antibodies specific for the T7-epitope (1:10000; Novagen) or the generated ZIP3 immunesera from rabbit $(1: 100)$ or guinea pig (1:100) and the enhanced chemoluminescence system (Amersham, Braunschweig, Germany). In blocking experiments, $10 \mu \mathrm{l}$ of the ZIP3 immunesera were preincubated with $5 \mu \mathrm{g}$ of the antigen.

\section{Cell culture}

For expression of ZIP proteins (rat) in human embryonic kidney (HEK-293) cells, T7-tagged coding sequences of ZIP1 and ZIP3 (see above) were inserted in the eukaryotic expression vector pBK/RSV (Stratagene, La Jolla, CA). HEK-293 cells were grown on poly(L-lysine) coated glass coverslips to approximately $50 \%$ confluency, transfected for $18 \mathrm{~h}$ with $1 \mu \mathrm{g}$ DNA using lipofection, washed, fixed and blocked for antibody stainings as described [14]. Subsequently, cells were incubated for $2 \mathrm{~h}$ with antibodies specific for the T7-epitope (1:1000; Novagen) or the ZIP3 immunesera from rabbit $(1: 100)$ or guinea pig $(1: 100)$. To visualize endogenous PKC- $\zeta$ present in HEK-293 cells, PKC- $\zeta$ specific antibodies were used (1:5000; Sigma-Aldrich, St. Louis, MO) After washing, the binding sites of the primary antibodies were revealed by the secondary antibodies Alexa ${ }^{\mathrm{TM}} 594$ (red fluorescence) and Alexa $^{\mathrm{TM}} 488$ (green fluorescence) goat anti-mouse, goat anti-rabbit or goat anti-guinea pig IgG $(\mathrm{H}+\mathrm{L})$ conjugates, (1:500; Molecular Probes, Eugene, OR). Blocking the primary antibodies was performed as described above. Fluorescent signals were examined by conventional light microscopy (DMIRB; Leica, Wetzlar, Germany).

\section{Immuneprecipitation}

Different organs, brain regions or 6 retinae of adult rats were homogenized on ice in buffer 1 (in $\mathrm{mM}$ : $150 \mathrm{NaCl} ; 5$ EDTA; 1 DTT; $20 \mathrm{Tris} / \mathrm{HCl} \mathrm{pH} 7.4$ including $1 \%$ Triton X-100) containing 25 units/ml Benzonase (Novagen) and protease inhibitors (Roche Diagnostics, Mannheim, Germany) using a glass/Teflon homogenizer. Thereafter, samples were centrifuged at $20.000 \times \mathrm{g}$ for $1 \mathrm{~h}$ at $4^{\circ} \mathrm{C}$. The supernatant was separated completely from the sediment and incubated for $18 \mathrm{~h}$ with $25 \mu \mathrm{l}$ of a $50 \%(\mathrm{v} / \mathrm{v})$ suspension of protein A-Agarose (Roche Diagnostics) that was preincubated with $50 \mu \mathrm{l}$ of the ZIP3 specific immuneserum from guinea pig. Beads were washed 4 times in $500 \mu \mathrm{l}$ buffer 2 (in mM: $150 \mathrm{NaCl} 5 \mathrm{EDTA}$; 50 Tris $/ \mathrm{HCl}$ pH 8 including $1 \%$ Triton X-100) and bound ZIP3 was detected on Western Blots using ZIP3 specific antibodies from rabbit (1:100). In control experiments, uncoated protein A-Agarose was used. For binding assays, HEK-293 cells were grown in $10 \mathrm{~cm}$ dishes and transfected with $5 \mu \mathrm{g}$ cDNA of T7-tagged ZIP3 from rat (see above) or FLAG-tagged ZIP3 from human inserted in pcDNA3.1 (Invitrogen, Carlsbad, CA) using lipofection. Proteins were precipitated with tag-specific antibodies, immobilized on protein A-Agarose as described above and bound PKC- $\zeta$ was detected on Western Blots using PKC- $\zeta$ specific antibodies (1:5000; Sigma-Aldrich). 


\section{Immunocytochemistry}

Vertical cryostat sections of the adult rat retinae were prepared as described [8] and incubated with primary antibodies recognizing ZIP3 (1:100; from guinea pig generated in this study), all 3 ZIP isoforms (1:100; PM045 from MBL, Woburn, MA), PKC- $\zeta$ (1:10000; SigmaAldrich) and PKC- $\alpha$ (1:100; Dunn Labortechnik, Asbach, Germany) to label rod bipolar cells. In addition, retina sections were co-stained with DAPI (1:1000; Sigma-Aldrich). The binding sites of the primary antibodies were revealed by secondary antibodies as described above. In controls, the primary antibodies were omitted which resulted in a complete loss of specific immunereactivity (not shown). Labelled proteins were analyzed by conventional light microscopy as above. Alternatively, Z-stacks of optical sections were taken with a Zeiss Axio Imager Z1 equipped with an ApoTome and projections were calculated with AxioVision 4.6.3 software (Zeiss, Oberkochen, Germany).

\section{RESULTS \\ Intronic polyadenylation signals are present in rat and human ZIP genes}

ZIP1 contains a PB1 domain, a ZZ zinc-finger, linear binding motifs for TRAF6, LC3 and Keap1, a serine/threonine rich region and an UBA domain for protein-protein interaction (Fig. $1 A$ ). Previously, we identified a new ZIP variant (ZIP3) in the rat CNS which contains an alternative C-terminal sequence of 13 amino acids. This sequence is generated by maintaining the intronic sequence between exon 4 and 5 of the rat gene (Fig. 1B). As a consequence, the open reading frame of exon 4 continues into intron 4 where it reaches a stop codon after 38 base pairs (Fig. 1C). Thus, ZIP3 contains the N-terminal PB1 and ZZ zinc-finger domains present in ZIP1/2, but lacks all interaction modules C-terminal of exon 4. Here, we identified a polyadenylation signal located about 120 bp $3^{\prime}$ of the stop codon in intron 4 (Fig. 1C). This signal is indeed used to add a poly(A) tail, as has been already shown by sequencing of the ZIP3 cDNA derived from rat brain (NCBI Accession number AF439403).

Next, we asked if short ZIP3-like isoforms are present in other mammals. To this end, we compared the sequences of intron 4 between rat, mouse and human (Fig. 1C). Besides conserved splice acceptor and donor sites, intronic sequences adjacent to the 3 ' end of exon 4 are different, resulting in different C-termini of potential ZIP3-like proteins in mouse and man. The human intron 4 carries a polyadenylation signal close to exon 5 , but no such signal was found in the mouse sequence (Fig. 1C). Based on these observations, we propose that short ZIP isoform similar to ZIP3 may be generated in rat and human by maintaining intron 4 which results in the truncation and polyadenylation of the ZIP transcript $5^{\prime}$ of exon 5.

\section{Detection of transcripts encoding for ZIP3-like variants in rat and human}

To test the above described predictions, mRNA from rat and mouse brains and from human HEK-293 cells was reversed transcribed for PCR analysis. The primer pairs P1/P2 amplified sequences located between exons 3 and 5 (Fig. 2A; Tab. 1). Resulting PCR products of $333 \mathrm{bp}$ in the rat and of comparable sizes in mouse and human represent the completely processed mRNA for ZIP1, containing exons 3, 4 and 5 (Fig. 2B). Other possible exon/intron combinations resulting in PCR products of larger size, as indicated on the left side of Fig. $2 \mathrm{~B}$, were not detected. These data indicate that ZIP transcripts containing exons 3 and 5 are complete spliced.

To detect intron 4 containing transcripts, we generated new antisense primers (P2i), located between predicted stop codons and polyadenylation signals of intronic sequences (Fig. 2A, Tab. 1). The primer combinations P1/P2i generated different PCR products in the 3 species (Fig. 2B). A signal of $348 \mathrm{bp}$ corresponded to the described ZIP3 isoform in rat, containing 
exons 3 and 4, followed by intron 4 that encodes for the ZIP3 specific C-terminal 13 amino acids (see Fig. 1C). A PCR product of comparable size with the same exon/intron composition was also present in human (Fig. 2B). In addition, in human we detected a PCR product of larger size (450 bp) that contained the complete intron 3 and part of intron 4 . Interestingly, maintenance of intron 3 does not induce a frame shift between exons 3 and 4 , pointing towards the possibility of a new ZIP isoform in humans. In contrast to rat and human, we could not detect any PCR product containing intronic sequences in the mouse, consistent with the absence of a polyadenylation signal in intron 4 of the mouse (see Fig. 1C). Thus, while ZIP1 is expressed in all species analyzed, only rat and human synthesize transcripts that encode for short ZIP3-like proteins.

Amino acid sequences encoded by ZIP3-like transcripts that contain intron 4 are $91 \%$ identical and $95 \%$ homologous between rat and human orthologues. While the C-terminal sequences encoded by intron 4 are very different between both species (see. Fig. 1C), the PKC- $\zeta$ binding PB1 domain shows a homology of $93 \%$. Since the rat ZIP3 protein interacts with PKC- $\zeta$ [8], we tested if the human ZIP3 counterpart binds PKC- $\zeta$, too. As a source for native PKC- $\zeta$ we used HEK-293 cells (Fig. 2C, left panel). Sequences encoding for rat and human ZIP3 were N-terminally tagged and expressed in HEK-293 cells. Binding of PKC- $\zeta$ was detected by immuneprecipitating ZIP3 proteins with tag-specific antibodies and subsequent visualization of the bound kinase by Western Blotting (Fig. 2C, right panels).

\section{Generation of ZIP3 specific antibodies}

In order to demonstrate that the observed ZIP3 transcript of rat is indeed translated into protein, we generated ZIP3 specific antibodies using the 13 rat specific C-terminal amino acids as immunogene (Fig. 3A). Obtained immunesera from rabbit and guinea pig were tested for their specificity with E.coli lysates containing recombinantly expressed ZIP proteins on denaturating Western Blots. Staining against N-terminally fused T7-epitopes ensured that comparable amounts of ZIP1, ZIP2 and ZIP3 were analyzed (Fig. 3B, arrowheads). Incubation of the Blots with the newly generated immunesera stained proteins of the predicted size for ZIP3 of $26 \mathrm{kDa}$ (Fig. 3C, D, black arrowheads). Specificity of the immunesera for ZIP3 was demonstrated by the absence of signals corresponding to ZIP1 or ZIP2 and by blocking the ZIP3 specific signals after pre-incubating the antibodies with the used peptide (Fig. 3C, D, white arrowheads). While the immuneserum derived from guinea pig recognized specifically ZIP3, the rabbit antibodies produced additional signals of higher molecular mass. Thus, for the subsequent detection of ZIP3 in the CNS we used the antibodies derived from guinea pig.

Before, we ensured that the generated immunesera would recognize the native ZIP3 protein. T7-tagged ZIP1 and ZIP3 constructs were expressed in HEK-293 cells and protein expression was monitored using T7-specific antibodies (Fig.3E, F; left panels). ZIP2 was not included because of its high similarity to ZIP1 and because the ZIP3 immunesera did not cross-react with ZIP2 on Western Blots. While ZIP1 appeared in intracellular speckles that are commonly described in the literature [3], ZIP3 had a more smooth cytoplasmic appearance. Incubation of ZIP1 expressing cells with the generated ZIP3 immunesera resulted in no detectable immuneflourescence, again demonstrating the specificity of the ZIP3 antibodies (Fig. 3E, F, upper panels). In contrast, incubation of ZIP3 expressing cells with the generated ZIP3 immunesera showed a perfect overlap between the T7 control stainings and the ZIP3 specific immuneflourescence (Fig. 3E, F, arrowhaeds in middle panels). These signals could be completely blocked by pre-incubating the antibodies with the ZIP3 C-terminal peptide (Fig. 3E, F, lower panels).

\section{ZIP3 is expressed in the central nervous system}

To analyze the expression of ZIP3 in the CNS, cytosolic proteins of a rat brain were homogenized and analyzed on a Western Blot using the generated ZIP3 immuneserum from guinea pig. Because no specific signal in the expected size of about $26 \mathrm{kDa}$ was detected (not shown), we enriched ZIP3 by immuneprecipitation with the guinea pig ZIP3 antibodies, 
analyzed precipitated proteins on Western Blots using the ZIP3 antibodies derived from rabbit and observed a strong ZIP3 positive signal (Fig. 4A, Brain). Besides being expressed in brain, we found high concentrations of ZIP3 in the liver and less protein in kidney, spleen and heart (Fig. 4A). Within the brain, ZIP3 was highest expressed in the cortex and cerebellum and lower in the other regions analyzed. In addition, we detected ZIP3 in the retina (Fig. 4A, Retina)

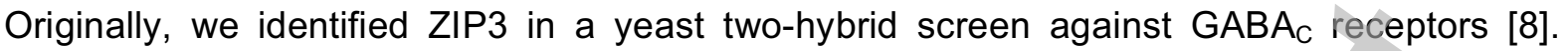
Because $\mathrm{GABA}_{C}$ receptors are highly expressed in the retina [15-17], we analyzed the subcellular localization of ZIP3 in this tissue. Staining of a vertical cryostat section of the rat retina with ZIP3 specific antibodies labelled cell bodies located in the inner nuclear layer (INL) and in the ganglion cell layer (GCL; Fig. 4B, asterisks in the middle panel). In contrast, the two synaptic layers (OPL and IPL) showed weaker and diffuse ZIP3 immunereactivity. Weak staining was also observed in the segments of photoreceptors (PRS), but not in their cell bodies that are located in the outer nuclear layer (ONL). Specificity of the label was ensured by pre-incubating the antibodies with the ZIP3 peptide (Fig. 4B, right panel).

Next, we compared the ZIP3 staining with the expression of other ZIP proteins, using antipan-ZIP antibodies recognizing amino acids $120-440$ of the human ZIP1. This epitope is about $90 \%$ identical to the corresponding rat sequence and includes the $Z Z$ zinc-finger domain present in exon 3 and C-terminal adjacent amino acids encoded by exon 4 that are identical between ZIP1 and ZIP3. Retinal cryostat sections were double-labelled with antibodies binding to all ZIP proteins and to ZIP3 only and co-localization of the stained proteins was analyzed in ApoTome generated optical sections. While both immunesera labelled cell bodies in the INL and GCL (Fig. 5A, asterisks), the anti-pan-ZIP antibodies showed also intense staining in the two synaptic layers (OPL and IPL). ZIP3 interacted with PKC- $\zeta$ [8] and indeed we observed co-localization of both proteins in cell bodies of the INL and GCL (Fig. 5B, asterisks). From these data, we conclude that ZIP3 is expressed in somata of the INL and GCL where it co-localizes with PKC- $\zeta$, while ZIP1/2 are also present in synaptic layers of the retina.

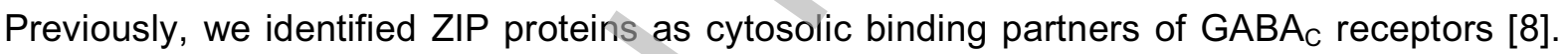
In the retina, $G A B A_{C}$ receptors are clustered at axon terminals of bipolar cells connecting rod photoreceptors, where they co-localize with PKC- $\zeta[8,15]$. Rod bipolar cells can be visualized with antibodies directed against PKC- $\alpha$ that label brightly their axon terminals located in the inner most part of the IPL, adjacent to the GCL (Fig. 6A). Applying the ZIP3 specific antibodies clearly demonstrated that ZIP3 is not present in rod bipolar cell axon terminals (Fig. 6B). In contrast, repeating the experiment with the anti-pan-ZIP antibodies revealed a clear expression of ZIP proteins in the PKC- $\alpha$ positive terminals in ApoTome generated optical sections, resulting in yellow colour in the double exposure (Fig. 6C, merge). This co-localization can be best seen when the yellow colour is selectively visualized in white (Fig. 6C, co-localization). In addition, higher power views of the boxed areas demonstrate the presence of ZIP proteins in the axon terminal systems of rod bipolar cells (Fig. 6C, lower panels). Thus, while ZIP3 is not at the correct sub-cellular location to interact with $\mathrm{GABA}_{C}$ receptors in the retina, other ZIP variants are expressed in rod bipolar cell axon terminals that also contain $\mathrm{GABA}_{C}$ receptors and $\mathrm{PKC}-\zeta$.

\section{DISCUSSION}

Alternative splicing is a major cellular mechanism that increases the diversity of proteins. It has been estimated that about $95 \%$ of all transcripts are alternatively spliced in humans [18]. Consequently, errors of the splicing machinery can lead to several diseases, including cystic fibrosis, spinal muscular atrophy, Duchenne muscular Dystrophy and tauopathies such as FTDP-17 (frontotemporal dementia with Parkinsonism linked to chromosome 17) [19]. The generation of protein isoforms is especially suited for a precise regulation of intracellular 
signal cascades, both in space and in time. One possibility to control the activity of simultaneous but distinct signal pathways is the use of scaffold proteins that physically assemble functionally related proteins into larger signal complexes. Protein kinase $\mathrm{C}-\zeta$ interacting proteins (ZIP1-3) are such scaffolds that function as multidomain protein adaptors and ensure the close vicinity of the enzymatic activity of the atypical protein kinases PKC- $\lambda / \mathrm{l}$ and PKC- $\zeta$ to target proteins.

Three ZIP isoforms have been described in the rat [8,9]. ZIP1 uses exons 1 to 8 of the ZIP gene, while ZIP2 and ZIP3 are both generated by variations at the $3^{\prime}$ end of exon 4. While ZIP2 lacks exon 5, in ZIP3 the intron between exon 4 and 5 is maintained and gives rise to 13 C-terminal amino acids. Importantly, this intron also contains an alternative polyadenylation signal and both, the respective polyadenylated transcript and the protein are expressed in the rat (see this study and NCBI Accession number AF439403 derived from [8]). It has been suggested that a dynamic interplay between alternative splicing and polyadenylation results in intronic polyadenylation events, which increases the complexity of the transcriptome [13]. Reported examples for the use of intronic polyadenylation sites include the genes of the immunegobulin $M$ heavy chain and the calcitonin gene-related peptide. The database for alternative splicing and transcript diversity (www.ebi.ac.uk/astd) currently lists about 7600 genes in human, 4300 genes in mouse and 1200 genes in rat that show both, splicing events and multiple polyadenylation signals [20].

As in rat, also in human we observed maintenance of intron 4 . The human ZIP gene contains a polyadenylation signal in this intron, which is located more downstream compared to the rat gene. Because the sequence of intron 4 differs between rat and human, a human specific sequence of 9 amino acids forms the predicted ZIP3-like C-terminus. In addition, in human we detected a transcript that contained intron 3. Inclusion of this intron preserves the open reading frame between exons 3 and 4, indicating a possible expression of a new ZIP isoform. At the moment, the presence of human proteins translated from transcripts containing introns 3 or 4 is unknown due to the lack of specific antibodies for the potential isoform-specific sequences. In contrast to rat and human, in the mouse we did not detect any polyadenylation signal in intron 4 and consistently, we were unable to amplify any PCR product containing sequences of the respective intron.

ZIP proteins contact PKC- $\zeta$ via the PB1 domain and $G A B A_{C}$ receptors via the $Z Z$ zinc-finger domain and indeed, simultaneous binding of PKC- $\zeta$ and $\mathrm{GABA}_{C}$ receptors to ZIP3 has been shown [8]. The PB1 and ZZ zinc-finger domains are encoded by exons 1 to 4 that are identical between ZIP1-3. Therefore, all 3 ZIP isoforms have the potential to target the kinase activity to the GABA receptor. In the retina, the anti-pan-ZIP antibodies, but not the ZIP3 specific immuneserum stained axon terminals of retinal bipolar cells that also express PKC- $\zeta$ and $\mathrm{GABA}_{C}$ receptors [8]. Thus, ZIP variants other than ZIP3 are expressed at the right place to anchor $P K C-\zeta$ to $G_{A B A}$ receptors. Several groups demonstrated phosphorylation of $\mathrm{GABA}_{C}$ receptors $\rho$-subunits, both in-vivo and in-vitro $[21,22]$. In this context it is noteworthy that protein phosphatase 1 , possibly counteracting the kinase activity, is anchored to $\mathrm{GABA}_{c}$ receptors by PNUTS [14]. Thus, our findings describe a potential molecular mechanism for the phosphorylation of $\mathrm{GABA}_{C}$ receptors.

We found ZIP3 localized in cell bodies of the retina that also express PKC- $\zeta$ but not GABA receptors. Thus, ZIP3 might serve different functions than ZIP1/2. Distinct ZIP isoforms might integrate the enzymatic activity of PKC- $\zeta$ in different signal pathways, thereby guiding enzymatic specificity by regulating the subcellular localizations of the kinase. This view is supported by the fact that PKC- $\zeta$ phosphorylated the interacting potassium channel $\mathrm{Kv} \beta 2$ with different efficiencies, depending on the presence of ZIP1 or ZIP2 [9]. 


\section{AUTHOR CONTRIBUTION}

Andreas Urbanczyk designed research and performed all experimental work, Anselm Jünemann obtained grant support, Ralf Enz obtained grant support, designed the research, supervised the study and wrote the paper. All authors contributed to the editing of the manuscript.

\section{ACKNOWLEDGMENTS}

We thank Stefan Hupfer for help with the preperation of rat organs, Kristina Tanneberger for providing FLAG-tag specific antibodies and Johann Helmut Brandstätter for retinal cryostat sections.

\section{FUNDING}

This work was supported by the Interdisciplinary Centre for Clinical Research (IZKF) at the university hospital of the Friedrich-Alexander-Universität Erlangen-Nürnberg. 


\section{REFERENCES}

1 Moscat, J., Diaz-Meco, M. T. and Wooten, M. W. (2007) Signal integration and diversification through the p62 scaffold protein. Trends Biochem. Sci. 32, 95-100

2 Geetha, T. and Wooten, M. W. (2002) Structure and functional properties of the ubiquitin binding protein p62. FEBS Lett. 512, 19-24

3 Moscat, J. and Diaz-Meco, M. T. (2009) p62 at the crossroads of autophagy, apoptosis, and cancer. Cell. 137, 1001-1004

4 Rodriguez, A., Duran, A., Selloum, M., Champy, M. F., Diez-Guerra, F. J., Flores, J. M., Serrano, M., Auwerx, J., Diaz-Meco, M. T. and Moscat, J. (2006) Mature-onset obesity and insulin resistance in mice deficient in the signaling adapter p62. Cell. Metab. 3, 211-222

5 Wooten, M. W., Hu, X., Babu, J. R., Seibenhener, M. L., Geetha, T., Paine, M. G. and Wooten, M. C. (2006) Signaling, polyubiquitination, trafficking, and inclusions: sequestosome 1/p62's role in neurodegenerative disease. J. Biomed. Biotechnol. 2006, 62079

6 Zatloukal, K., Stumptner, C., Fuchsbichler, A., Heid, H., Schnoelzer, M., Kenner, L., Kleinert, R., Prinz, M., Aguzzi, A. and Denk, H. (2002) p62 Is a common component of cytoplasmic inclusions in protein aggregation diseases. Am. J. Pathol. 160, 255-263

7 Ramesh Babu, J., Lamar Seibenhener, M., Peng, J., Strom, A. L., Kemppainen, R., Cox, N., Zhu, H., Wooten, M. C., Diaz-Meco, M. T., Moscat, J. and Wooten, M. W. (2008) Genetic inactivation of p62 leads to accumulation of hyperphosphorylated tau and neurodegeneration. J. Neurochem. 106, 107-120

8 Croci, C., Brandstätter, J. H. and Enz, R. (2003) ZIP3, a new splice variant of the PKC-zetainteracting protein family, binds to GABAC receptors, PKC-zeta, and Kv beta 2. J. Biol. Chem. 278, 6128-6135

9 Gong, J., Xu, J., Bezanilla, M., van Huizen, R., Derin, R. and Li, M. (1999) Differential stimulation of PKC phosphorylation of potassium channels by ZIP1 and ZIP2. Science. 285, 1565-1569

10 Kim, O. J., Ariano, M. A., Namkung, Y., Marinec, P., Kim, E., Han, J. and Sibley, D. R. (2008) D2 dopamine receptor expression and trafficking is regulated through direct interactions with ZIP. J. Neurochem. 106, 83-95

11 Moscat, J., Diaz-Meco, M. T. and Wooten, M. W. (2009) Of the atypical PKCs, Par-4 and p62: recent understandings of the biology and pathology of a PB1-dominated complex. Cell. Death Differ. 16, 1426-1437

12 Puls, A., Schmidt, S., Grawe, F. and Stabel, S. (1997) Interaction of protein kinase C zeta with ZIP, a novel protein kinase C-binding protein. Proc. Natl. Acad. Sci. U S A. 94, 6191-6196

13 Tian, B., Pan, Z. and Lee, J. Y. (2007) Widespread mRNA polyadenylation events in introns indicate dynamic interplay between polyadenylation and splicing. Genome Res. 17, 156-165

14 Rose, M., Dütting, E., Schröder, N., Sticht, H., Brandstätter, J. H. and Enz, R. (2008) PNUTS forms a trimeric protein complex with $\mathrm{GABA}(\mathrm{C})$ receptors and protein phosphatase 1 . Mol. Cell. Neurosci. 37, 808-819

15 Enz, R., Brandstätter, J. H., Wässle, H. and Bormann, J. (1996) Immunocytochemical localization of the GABAc receptor rho subunits in the mammalian retina. J. Neurosci. 16, 4479-4490

16 Enz, R. and Cutting, G. R. (1999) GABAC receptor rho subunits are heterogeneously expressed in the human CNS and form homo- and heterooligomers with distinct physical properties. Eur. J. Neurosci. 11, 41-50

17 Enz, R., Brandstätter, J. H., Hartveit, E., Wässle, H. and Bormann, J. (1995) Expression of GABA receptor rho 1 and rho 2 subunits in the retina and brain of the rat. Eur. J. Neurosci. 7, 14951501

18 Pan, Q., Shai, O., Lee, L. J., Frey, B. J. and Blencowe, B. J. (2008) Deep surveying of alternative splicing complexity in the human transcriptome by high-throughput sequencing. Nat. Genet. 40, 1413-1415

19 Cooper, T. A., Wan, L. and Dreyfuss, G. (2009) RNA and disease. Cell. 136, 777-793

20 Koscielny, G., Le Texier, V., Gopalakrishnan, C., Kumanduri, V., Riethoven, J. J., Nardone, F., Stanley, E., Fallsehr, C., Hofmann, O., Kull, M., Harrington, E., Boue, S., Eyras, E., Plass, M., Lopez, F., Ritchie, W., Moucadel, V., Ara, T., Pospisil, H., Herrmann, A., J, G. R., Guigo, R., Bork, P., Doeberitz, M. K., Vilo, J., Hide, W., Apweiler, R., Thanaraj, T. A. and Gautheret, D. (2009) ASTD: The Alternative Splicing and Transcript Diversity database. Genomics. 93, 213220

21 Feigenspan, A. and Bormann, J. (1994) Differential pharmacology of GABAA and GABAC receptors on rat retinal bipolar cells. Eur. J. Pharmacol. 288, 97-104

22 Sedelnikova, A. and Weiss, D. S. (2002) Phosphorylation of the recombinant rho1 GABA receptor. Int. J. Dev. Neurosci. 20, 237-246 
Table 1. DNA sequence of oligonucleotides used to analyze splicing of ZIP transcripts, given in the direction from 5' to 3'. The GAPDH specific primers recognize the corresponding transcripts in all species indicated. (P1 - sense primer; P2 or P2i antisense primers)

\begin{tabular}{|l|l|l|l|}
\hline & rat & mouse & human \\
\hline P1 & GCCTGTGGTGGGAACTCG & CAACGGGCCTGTGGTGG & GCCTGTGGTAGGAACCCG \\
\hline P2 & ACGCTCTCCCCCACATTC & CACACTCTCCCCCACATTC & CACACTCTCCCCAACGTTC \\
\hline P2i & CTTTAGGCCAAGGGTCCAC & CTTGGCCAAGGGCCTACC & CACAAGGGCCCTGCACAC \\
\hline GAPDH & & & \\
\hline P1 & CTTCACCACCATGGAGAAGG & \\
\hline P2 & CCTGCTTCACCACCTTCTTG & & \\
\hline
\end{tabular}




\section{FIGURE LEGENDS}

Figure 1. Protein and gene structure of ZIP family members. (A) Domain organization of the rat ZIP1 protein. Protein-protein interaction sites are shown as black boxes (PB1 - Phox and Bem1p1 domain; ZZ - ZZ zinc-finger; T - TRAF6 binding sequence; S/T serine/threonine rich region; L - LC3 interacting region; KIR - Keap1-interacting region; UBA - ubiquitin associated domain). (B) Exon/intron structure of the rat ZIP gene and composition of ZIP1-3 transcripts. Exons are numbered (1 to 8 ) and are shown as white boxes, while introns are represented by horizontal lines. The dashed line indicates skipping of exon 5 which generates ZIP2. The dotted line marks the boundary between exon 4 and intron 4 , where the ZIP3 specific C-terminus is added. (C) The region between exons 4 and 5 of the rat ZIP gene, or corresponding sequences in mouse and human, are enlarged (thick line) with DNA sequences of exons and introns represented by letters in upper and lower case, respectively. Signals for the termination of protein biosynthesis and polyadenylation are highlighted in bold. Grey boxes indicate the localization of splice acceptor and donor sites. Protein sequences are given in the single letter code for amino acids. Underlined sequences encode for amino acids not present in ZIP1 or ZIP2. Stop codons are marked with an "\#". Relative sizes of exons, introns and protein domains are drawn to scale.

Figure 2. Detection of transcripts containing intronic sequences. (A) Detailed view of the ZIP gene structure between exons 3 and 5 from Fig. 1B. Thick lines resemble stop codons (tag) and polyadenylation signals (aataaa). The location of PCR primers is indicated by arrows. (P1 - sense primers; P2 or P2i - antisense primers). (B) Agarose gels showing DNA fragments obtained by RT-PCR from the indicated tissues (+ RT). In control samples the reverse transcriptase was omitted (- RT) which resulted in the absence of PCR products. PCR fragments of the house keeping gene GAPDH compared the cDNA concentrations between samples (lower panel). The molecular weight marker (pRK7/Hinfl) is indicated in base pairs on the right. The left side gives an overview of theoretical exon/intron combinations: Exons are numbered, horizontal lines represent introns and "\#" indicates stop codons. The expected lengths of PCR products is given in base pairs (bp) as calculated for the rat. Exon/intron combinations representing ZIP1 or ZIP3 are indicated in bold. (C) Endogenous PKC- $\zeta$ in HEK-293 cells was visualized by immunostaining (left panel, the scale bar is $10 \mu \mathrm{m}$ ). Rat and human ZIP3 proteins containing C-terminal sequences encoded by intron 4 were $\mathrm{N}$-terminally tagged, expressed in HEK-293 cells and immunoprecipitated (IP) with tag-specific antibodies. Co-precipitation of endogenous PKC- $\zeta$ was detected on Western Blots (right panels). The input controls for the expression of rat and human ZIP proteins.

Figure 3. Characterization of ZIP3 specific antibodies. (A) Domain organization of ZIP3 (abbreviations as in Fig. 1A). The peptide sequence used for antibody generation is shown in the single letter code with the 13 ZIP3 specific amino acids highlighted in bold. E.coli lysates containing recombinantly expressed T7-tagged ZIP proteins were analyzed on denaturating Western Blots with antibodies specific for the T7-epitope (B) or for ZIP3 derived from rabbit (C) or guinea pig (D). Pre-incubation of ZIP3 specific immunesera with the antigen inhibits the signal (blocked, white arrowheads). HEK-293 cells expressing T7-tagged ZIP1 or ZIP3 were labelled with ZIP3 specific antibodies derived from rabbit (E) or guinea pig (F) or with T7-specific antibodies for control (left panels). Arrowheads point to cytosolic regions that were double stained for the T7-tag and ZIP3. Pre-incubation of ZIP3 specific immunesera with the antigen resulted in a complete loss of fluorescence (blocked). The scale bar represents $10 \mu \mathrm{m}$.

Figure 4. ZIP3 specific antibodies detect endogenous ZIP3 protein in brain and retina. (A) ZIP3 specific antibodies derived from guinea pig were used to immuneprecipitate cytosolic proteins from the indicated tissues of adult rats. Precipitated ZIP3 was detected by Western Blotting using the rabbit ZIP3 antiserum (ZIP3). In negative controls the guinea pig ZIP3 specific antibodies were omitted (neg.). (B) Vertical cryostat sections through the adult 
rat retina incubated with DAPI to visualize retinal layers (cyan, left panel) or ZIP3 specific antibodies from guinea pig (green, middle panel). ZIP3 specific label on somata in the INL and GCL (asterisks) can be blocked by pre-incubation the antibodies with the peptide (right panel). (PRS - photoreceptor segments, ONL - outer nuclear layer, OPL - outer plexiform layer, INL - inner nuclear layer, IPL - inner plexiform layer, GCL - ganglion cell layer). The scale bar represents $20 \mu \mathrm{m}$.

Figure 5. ZIP3 and PKC- $\zeta$ are co-expressed in cell bodies of the rat retina. (A) ApoTome generated micrographs of a vertical section through the adult rat retina doublelabelled with antibodies specific for ZIP3 (guinea pig, green) and recognizing all ZIP isoforms (pan-ZIP, red). Co-stained cell bodies in the INL and GCL are marked with asterisks. For better visualization, the regions located between the horizontal dashed lines are enlarged and overlaid in the lower panel (merge). (B) Double labelling of a retinal section for ZIP3 and PKC- $\zeta$, visualized as in (A). Abbreviations as in Fig. 4B. Scale bars are $20 \mu \mathrm{m}$.

Figure 6. ZIP proteins are expressed in axon terminal systems of rod bipolar cells in the rat retina. (A) Rod bipolar cells are immunereactive for PKC- $\alpha$ (red). Their cell bodies are located in the outer part of the INL, their dendrites extend into the OPL and their axons terminate in the innermost region of the IPL close to somata in the GCL (cyan). (B) ApoTome generated micrographs of a vertical section through the adult rat retina doublelabelled for PKC- $\alpha$ (red) and ZIP3 (green). Enlargement of the regions between the dashed lines demonstrates that ZIP3 is not expressed on PKC- $\alpha$ labelled axon terminals (merge). (C) ApoTome generated micrographs of a retinal cryostat section double-labelled with a PKC- $\alpha$ specific immuneserum (red) and antibodies recognizing all ZIP isoforms (pan-ZIP, green). The regions indicated by dashed lines are enlarged and overlaid (merge). For clarity, resulting yellow signals are shown separately in white (co-localization). Higher power views of the boxed regions $(a, b)$ demonstrate the presence of ZIP proteins on axon terminals of rod bipolar cells. Abbreviations as in Fig. 4B. Scale bars are $20 \mu \mathrm{m}$. 


\section{Figure 1}

B

\begin{tabular}{l|l|l|l|l|l|l|l|l|}
\hline ZIP1 & 1 & 2 & 3 & 4 & 5 & 6 & 7 & 8 \\
\hline
\end{tabular}

ZIP3 \begin{tabular}{|l|l|l|l|l|}
\hline 1 & 2 & 3 & 4 & 4 \\
\hline
\end{tabular}

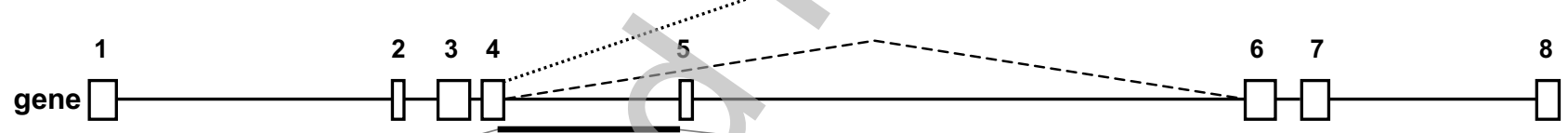

C) $\frac{\text { rat }}{\text { Exon } 4-S \quad G \quad K} A$ G $V$ C ... TCGGgtaaggctggtgtttgcactggctttaagtgtcataagtagcca...116bp...cataataaataa...1058bp...саgCTTCT ....

mouse

Exon 4-S G $\mathrm{K} \quad \mathrm{T} \quad \mathrm{G}$ I $\quad \# \quad$ A $\mathrm{S}$ - Exon 5

... TCAGgtaagactggtatttgaactagc ... 1030bp ... саgсTTCT ...

human

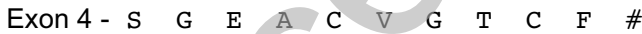

A $S$ - Exon 5

... TCAGgtgaggcttgtgttggaacctgcttctgattggtg ... 745bp ... ggcattaaagat ... 27bp ... aagCTTCT .... 


\section{Figure 2}

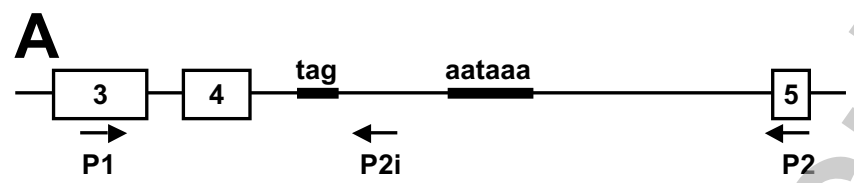

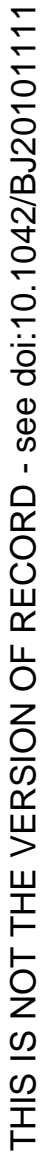
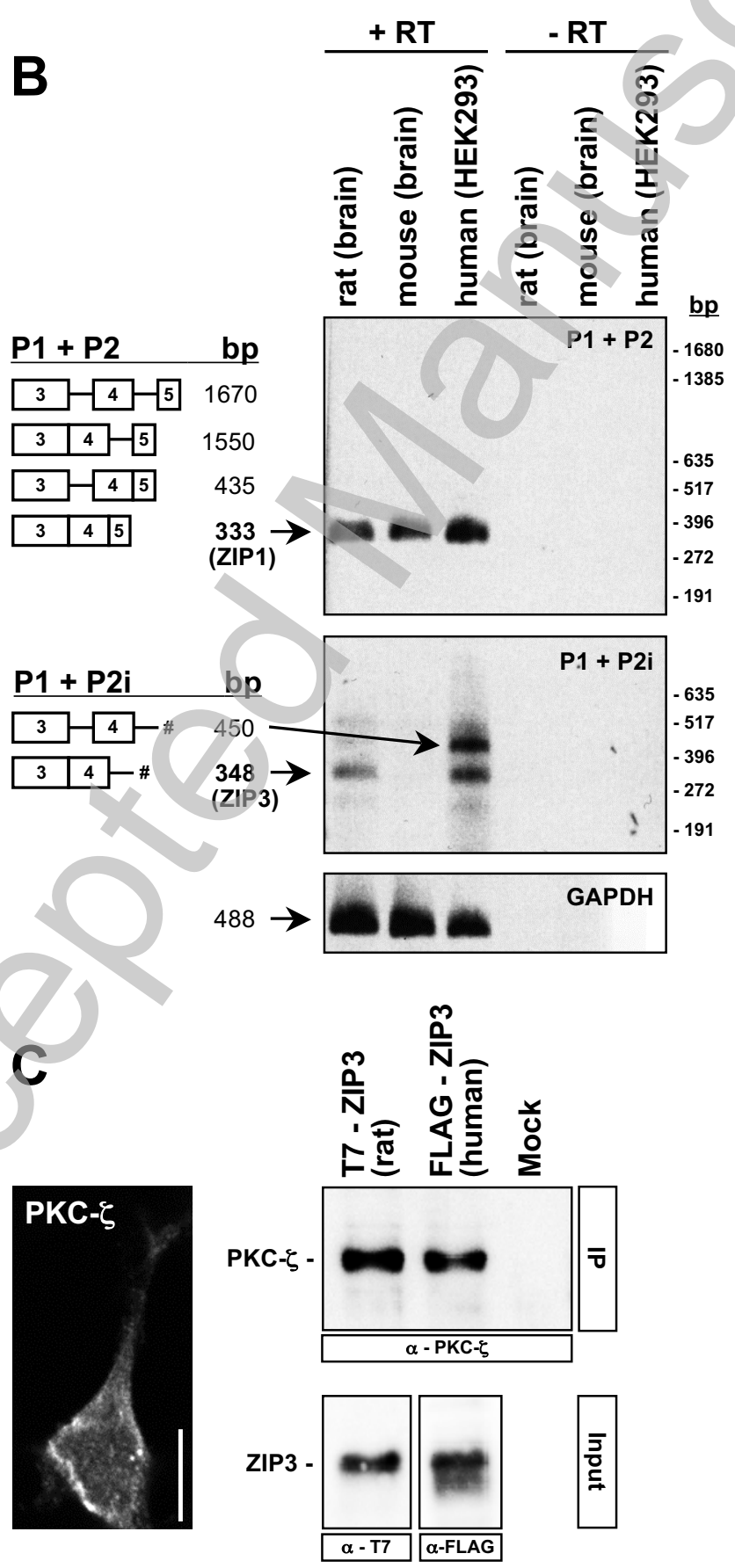

Licenced copy. Copying is not permitted, except with prior permission and as allowed by law. (C) 2010 The Authors Journal compilation (c) 2010 Portland Press Limited 
Biochemical Journal Immediate Publication. Published on 28 Oct 2010 as manuscript BJ20101111

\section{Figure 3}

\section{A}

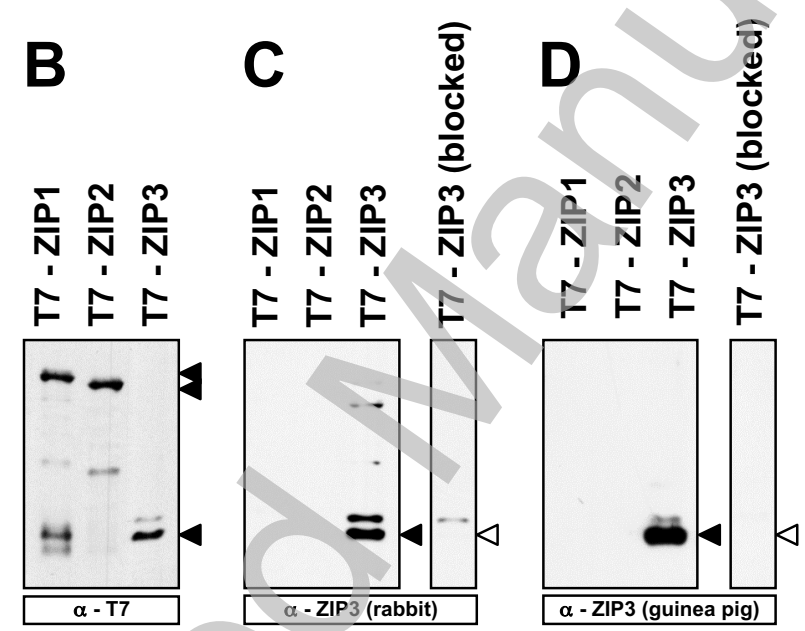

$\mathbf{E}$
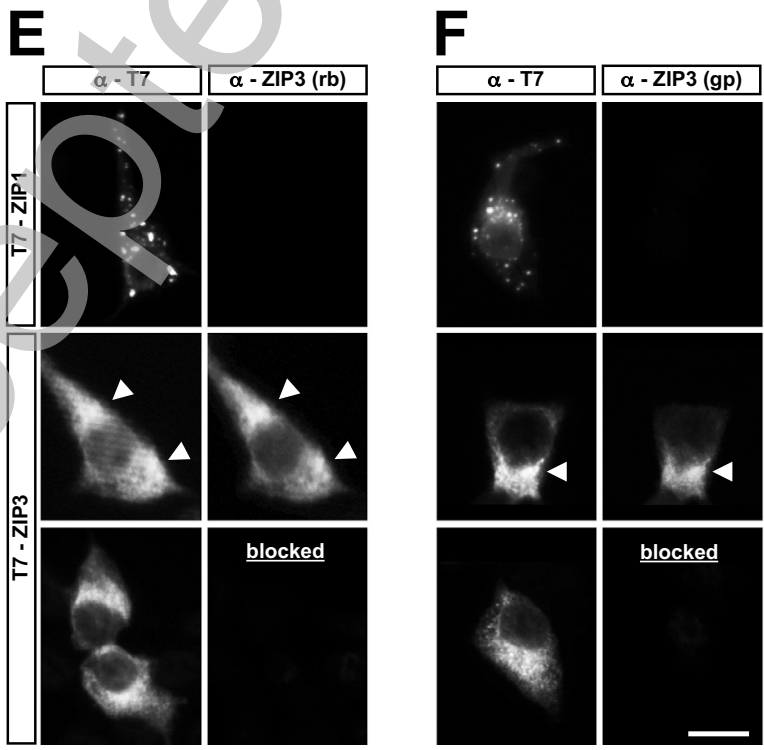

Licenced copy. Copying is not permitted, except with prior permission and as allowed by law. (c) 2010 The Authors Journal compilation (c) 2010 Portland Press Limited 
Biochemical Journal Immediate Publication. Published on 28 Oct 2010 as manuscript BJ20101111

\section{Figure 4}
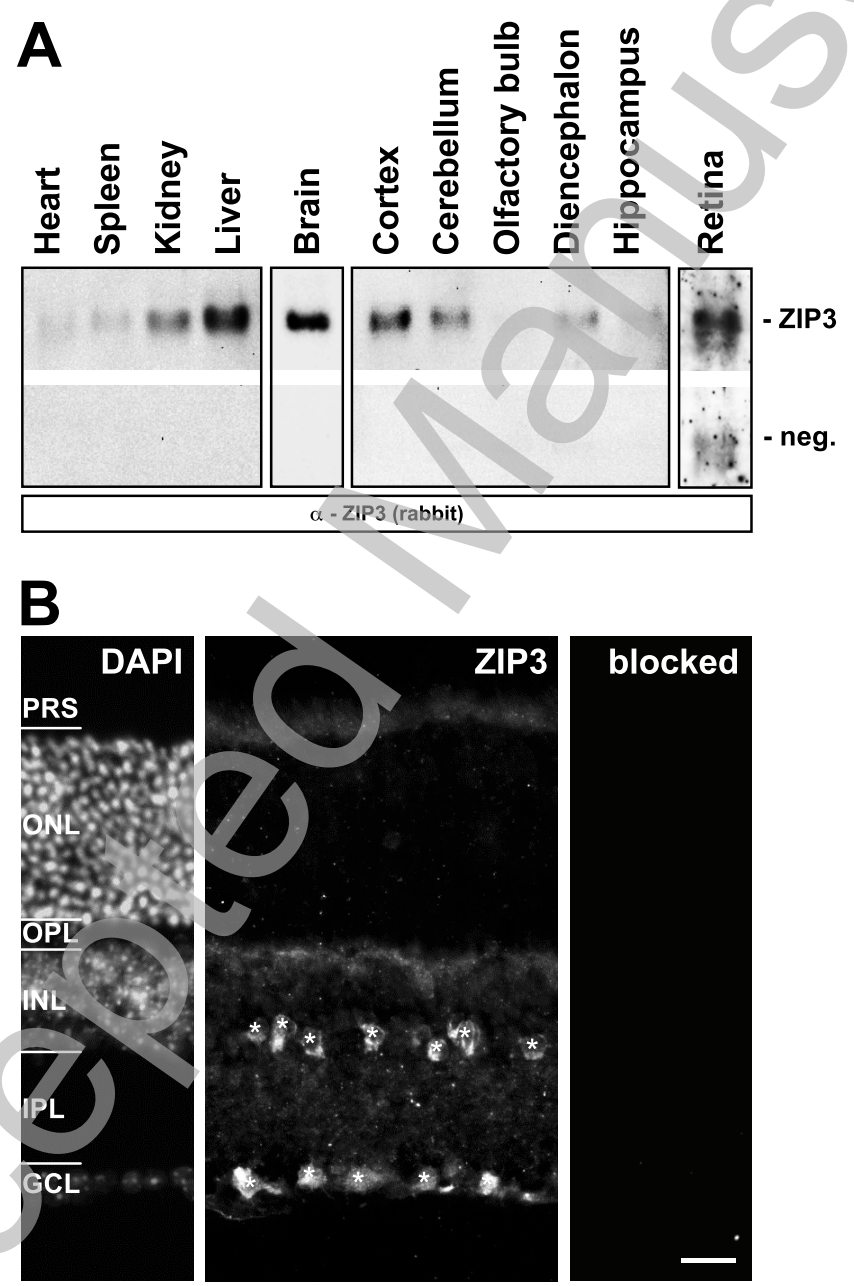
Biochemical Journal Immediate Publication. Published on 28 Oct 2010 as manuscript BJ20101111

\section{Figure 5}
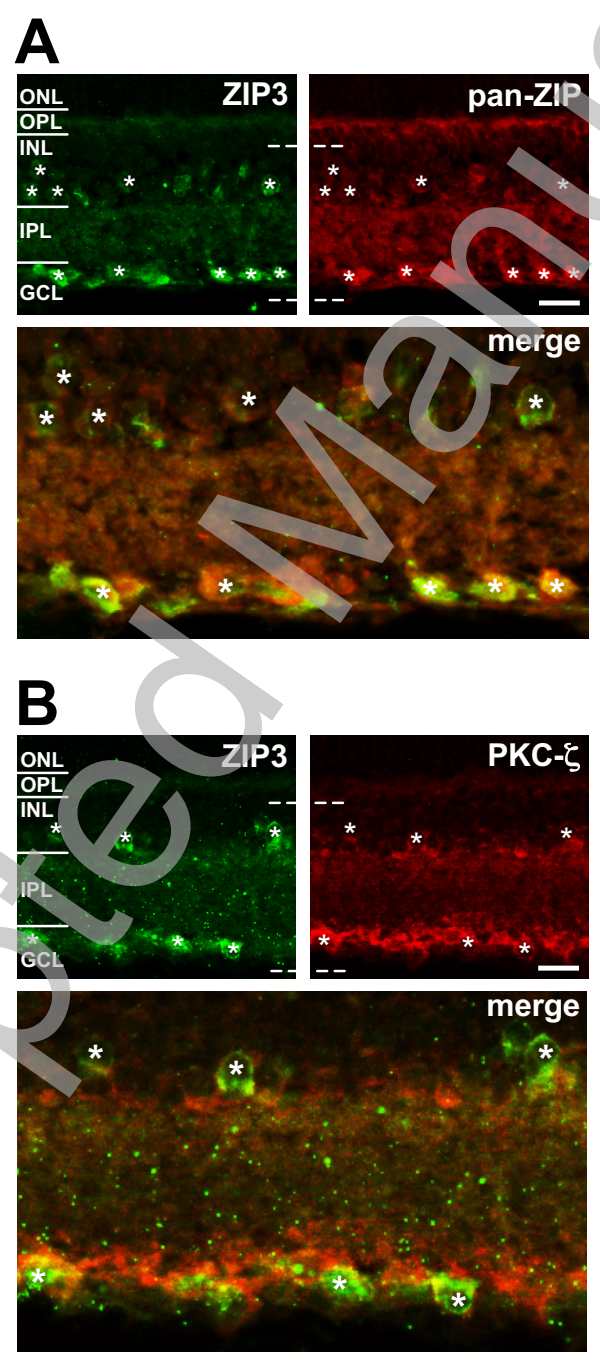
Biochemical Journal Immediate Publication. Published on 28 Oct 2010 as manuscript BJ20101111

\section{Figure 6}
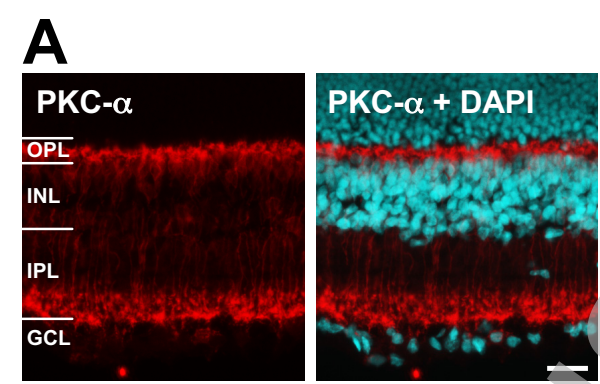

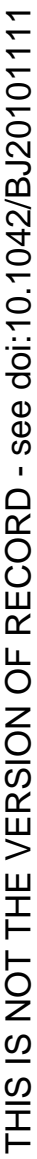
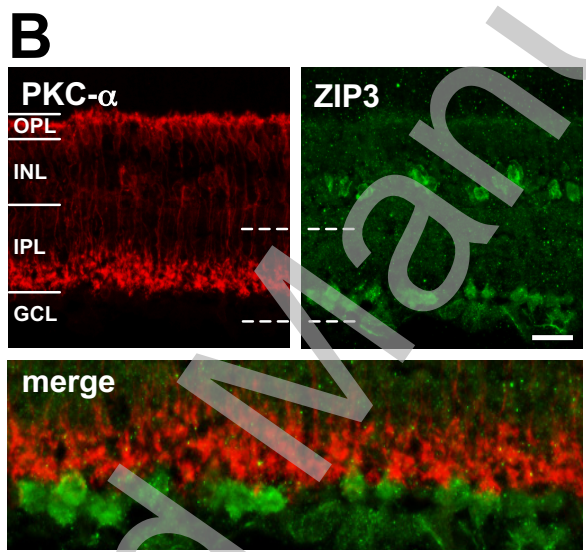

C

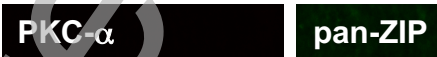

OPL
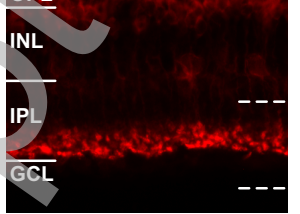

merge
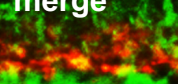

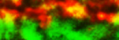

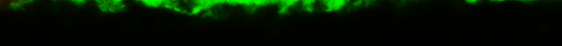

co-localization a
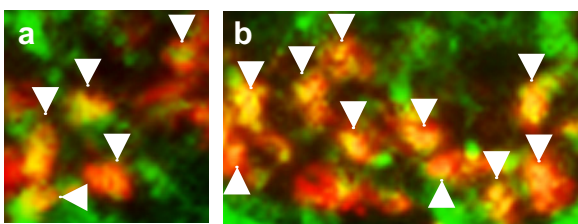

Licenced copy. Copying is not permitted, except with prior permission and as allowed by law. (c) 2010 The Authors Journal compilation (c) 2010 Portland Press Limited 